\title{
Ovarian yolk sac tumor in a patient with sexual differentiation disorder: a case description
}

\author{
Chao Lian Xie ${ }^{1}$, Chun Rong Peng ${ }^{2}$, Jia Xin Yan ${ }^{3}$, Lin Xia Wang ${ }^{4}$, Peng Zhou ${ }^{1}$ \\ ${ }^{1}$ Department of Radiology, Sichuan Cancer Hospital and Institute, Sichuan Cancer Center, School of Medicine, University of Electronic Science \\ and Technology of China, Chengdu, China; ${ }^{2}$ Department of Gynecologic Oncology, Sichuan Cancer Hospital and Institute, Sichuan Cancer Center, \\ School of Medicine, University of Electronic Science and Technology of China, Chengdu, China; ${ }^{3}$ Department of Pathology, Sichuan Cancer \\ Hospital and Institute, Sichuan Cancer Center, School of Medicine, University of Electronic Science and Technology of China, Chengdu, China; \\ ${ }^{4}$ Department of Radiology, Chengdu First People's Hospital, Chengdu Integrated Traditional and Western Medicine Hospital, Chengdu, China
}

Correspondence to: Peng Zhou. Department of Radiology, Sichuan Cancer Hospital and Institute, Sichuan Cancer Center, School of Medicine, University of Electronic Science and Technology of China, Chengdu 610041, China. Email: penghyzhou@126.com.

Submitted Jul 21, 2020. Accepted for publication Dec 02, 2020.

doi: 10.21037/qims-20-890

View this article at: http://dx.doi.org/10.21037/qims-20-890

\section{Introduction}

Yolk sac tumor (YST), also called endodermal sinus tumor, is a rare malignant tumor accounting for less than $1 \%$ of all ovarian malignancies and is the second most common malignant ovarian germ cell tumor (MOGCT) $(1,2)$. Disorder of sexual differentiation (DSD), once known as hermaphroditism, is also a rare cause of intersexuality disorders, where an individual has both male and female gonads $(3,4)$. Due to the rarity of the condition, most articles about DSD are case reports and are limited in number $(3,5)$.

To the best of our knowledge, the YST of the ovary in adult DSD has rarely been reported in the literature. Here, we report a case of an adult DSD patient with an ovarian YST and their follow-ups after surgical treatment and chemotherapy.

\section{Case presentation}

A 23-year-old patient (the social sex was male, while the karyotype was $46, \mathrm{XX}$ ), with no previous medical and surgical history, consulted for abdominal pain, which was first noticed 1 month earlier and was aggravated with vomiting for 3 days.

\section{Physical examination}

A palpable hard mass approximately $8 \mathrm{~cm} * 8 \mathrm{~cm}$ in size located in the right lower abdomen was found, with poor activity and free of tenderness. The secondary sexual characteristics and external genitalia were males. However, the penis was short, the bilateral scrotum was empty, and the prostate was not touched by rectal finger examination. The bilateral inguinal areas were normal.

\section{Laboratory inspections}

The patient had an elevated alpha fetoprotein (AFP) level of $45,470 \mathrm{ng} / \mathrm{mL}$, a decreased testosterone level of $0.18 \mathrm{ng} / \mathrm{mL}$, and an estradiol level of $85.44 \mathrm{pg} / \mathrm{mL}$. The routine blood tests, biochemistry, coagulation, follicle stimulating hormones, and chorionic gonadotropin were normal.

\section{Magnetic resonance imaging (MRI)}

A huge mass, measuring approximately $18 \mathrm{~cm}^{*} 15 \mathrm{~cm}^{*} 9 \mathrm{~cm}$, was located in the lower abdomen on the pelvic cavity's left side. It mainly showed hypointensity with patchy slight hyperintensity on fat-suppressed T1-weighted images (Figure 1A) and inhomogeneous hyperintensity on fat- 

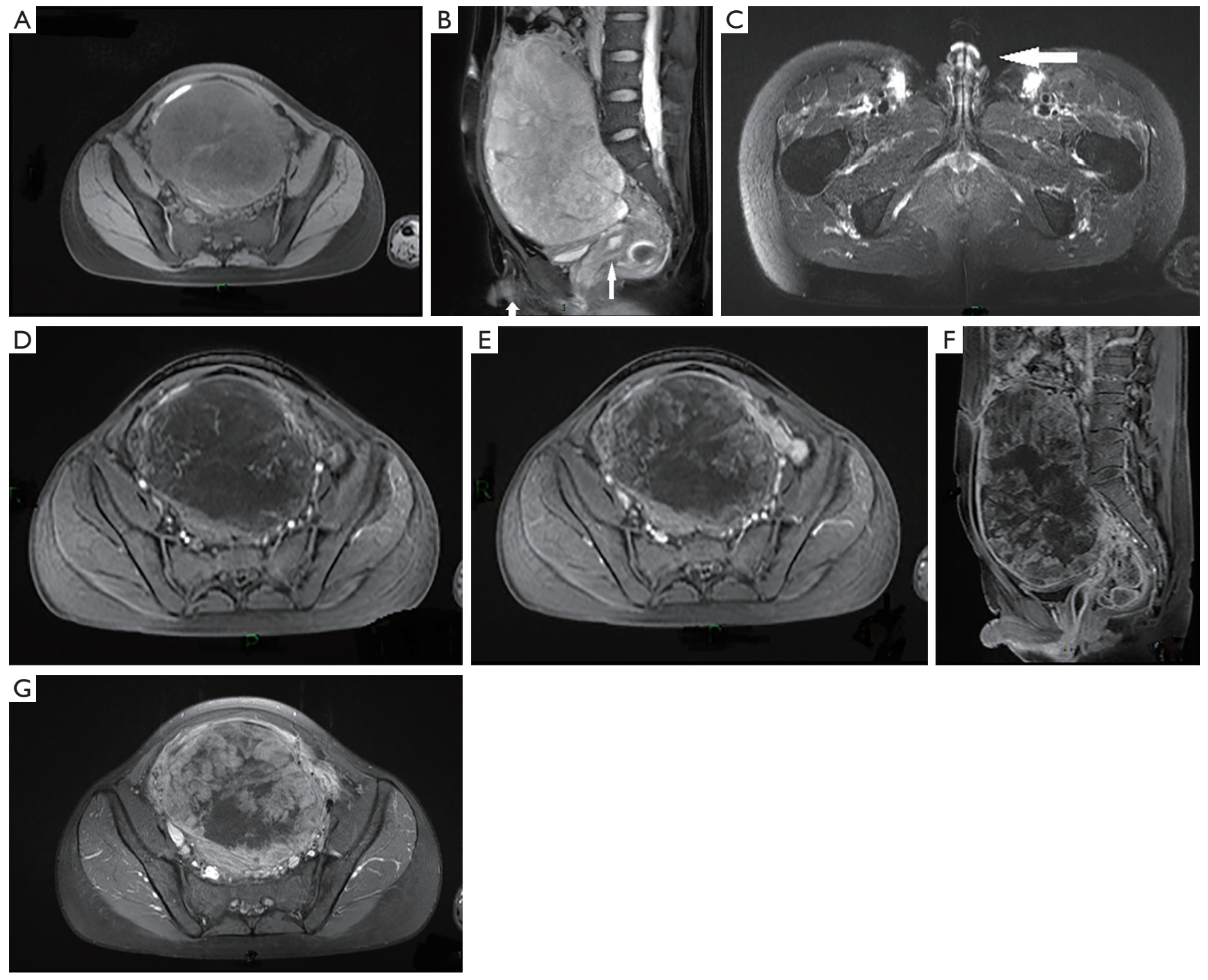

Figure 1 A 23-year-old patient with a huge mass in the lower abdomen and the left side of the pelvic cavity. The mass showed hypointensity with patchy slight hyperintensity on axial T1-weighted images (T1WI) with fat suppression (A) and inhomogeneous hyperintensity on sagittal T2-weighted images (T2WI) with fat suppression (B). Furthermore, there was a short penis and a small uterus in the pelvis (B,C, arrows), and no testicles in the scrotum (C, arrow). On contrast-enhanced images, it showed inhomogeneous mild enhancement in the arterial phase (D) and gradually significant heterogeneous enhancement in the venous (E), sagittal, and axial delayed phase (F,G).

suppressed T2-weighted images (Figure 1B). Also, there was a short penis and a small uterus in the pelvis but no testicles in the scrotum, and no prostate and no seminal vesicle gland in the pelvis (Figure 1B,C). On contrast-enhanced images, it showed mild inhomogeneous enhancement in the arterial phase (Figure 1D) and gradually significant heterogeneous enhancement in the venous (Figure $1 E$ ) and delayed phase (Figure 1F,G).

\section{Surgical findings}

The patient received resections of the mass, the left fallopian tube, part of the peritoneum and omentum, and the vermiform appendix under general anesthesia. The mass, approximately $17 \mathrm{~cm} * 12 \mathrm{~cm}^{*} 11 \mathrm{~cm}$ in size, was directly connected to the left fallopian tube and widely adhered to the surrounding tissue fascia. The left ovary was not clear. It involved the peritoneum, omentum, and 

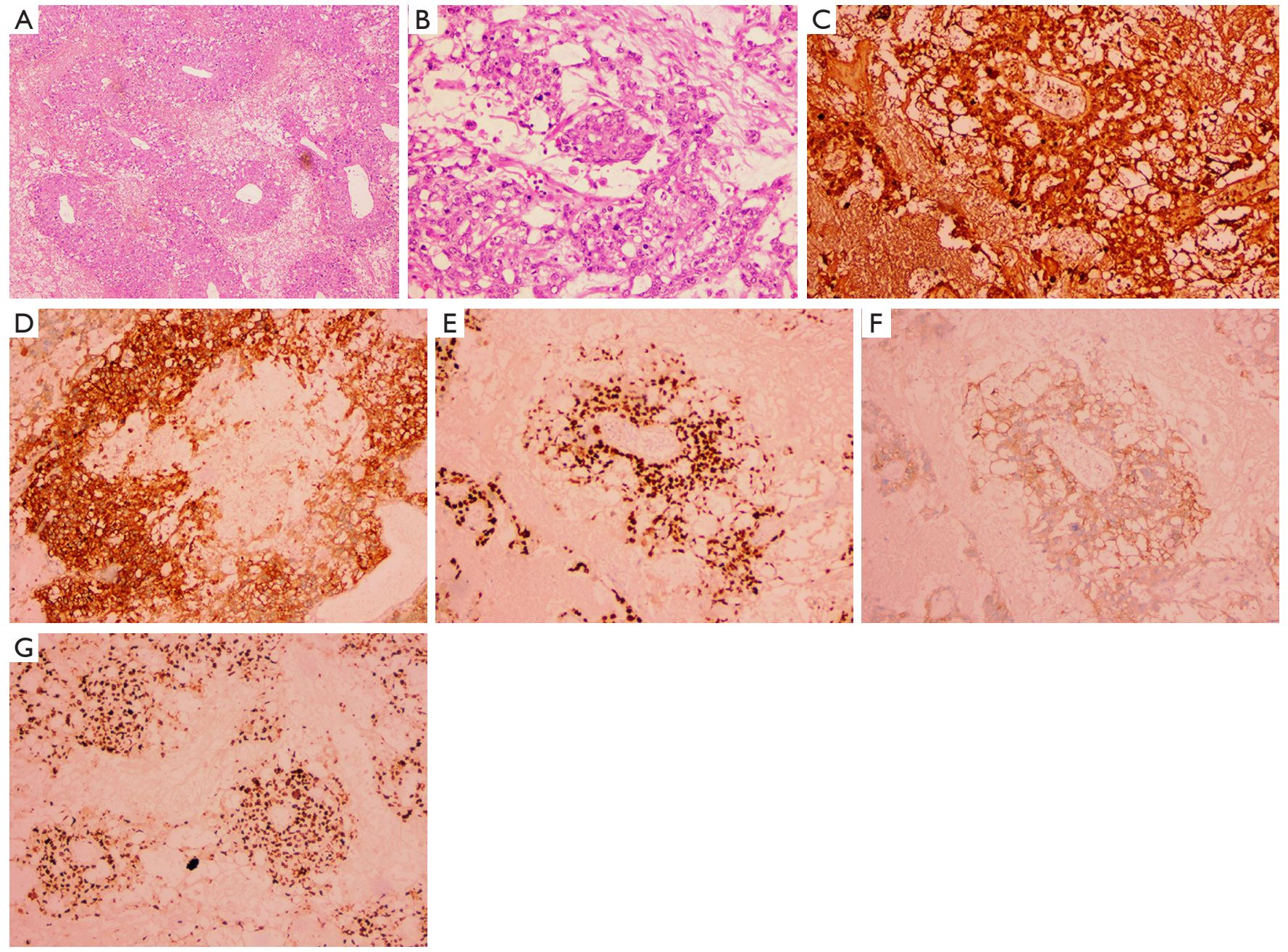

Figure 2 Hematoxylin-eosin (HE) staining showed scattered adjacent tube-like structures, surrounded by necrosis (A, HE $\times 40)$ and microcapsules, and cystic structural features $(\mathrm{B}, \mathrm{HE} \times 200)$. Immunohistochemistry was positive for alpha-fetoprotein $(\mathrm{AFP})(\mathrm{C})$, placental alkaline phosphatase (PLAP) (D), sal-like protein 4 (SALL4) (E), c-kit receptor (CD117) (F), and Ki67 antigen (Ki67, 80\%) (G). AFP, PLAP, SALL4, CD117, and Ki67 were all ×200.

appendix. The right side of the fallopian tube and ovary were visible and normal. The uterus was small.

\section{Histopathological findings}

The tumor was free of a capsule and had yellowish fishlike tissue with obvious bleeding and necrosis. The cut surface was slightly soft, appearing gray and white. The peritoneum and omentum were involved with the tumor. The left reproductive vessel and appendix were only infiltrated by inflammatory cells. Hematoxylin-eosin (HE) staining showed scattered adjacent tube-like structures, surrounded by necrosis and microcapsules, as well as cystic structural features (Figure 2A,B). Immunohistochemistry was positive for AFP, placental alkaline phosphatase (PLAP), sal-like protein 4 (SALL4), c-kit receptor (CD117), and Ki67 antigen (Ki67) 80\% (Figure 2C,D,E,F,G), and negative for CD30 antigen (CD30) and D2-40 antibody (D2-40). Combined with HE morphology, it supported the diagnosis of YST.

\section{Follow-up}

Two months after surgery, the patient was admitted with 

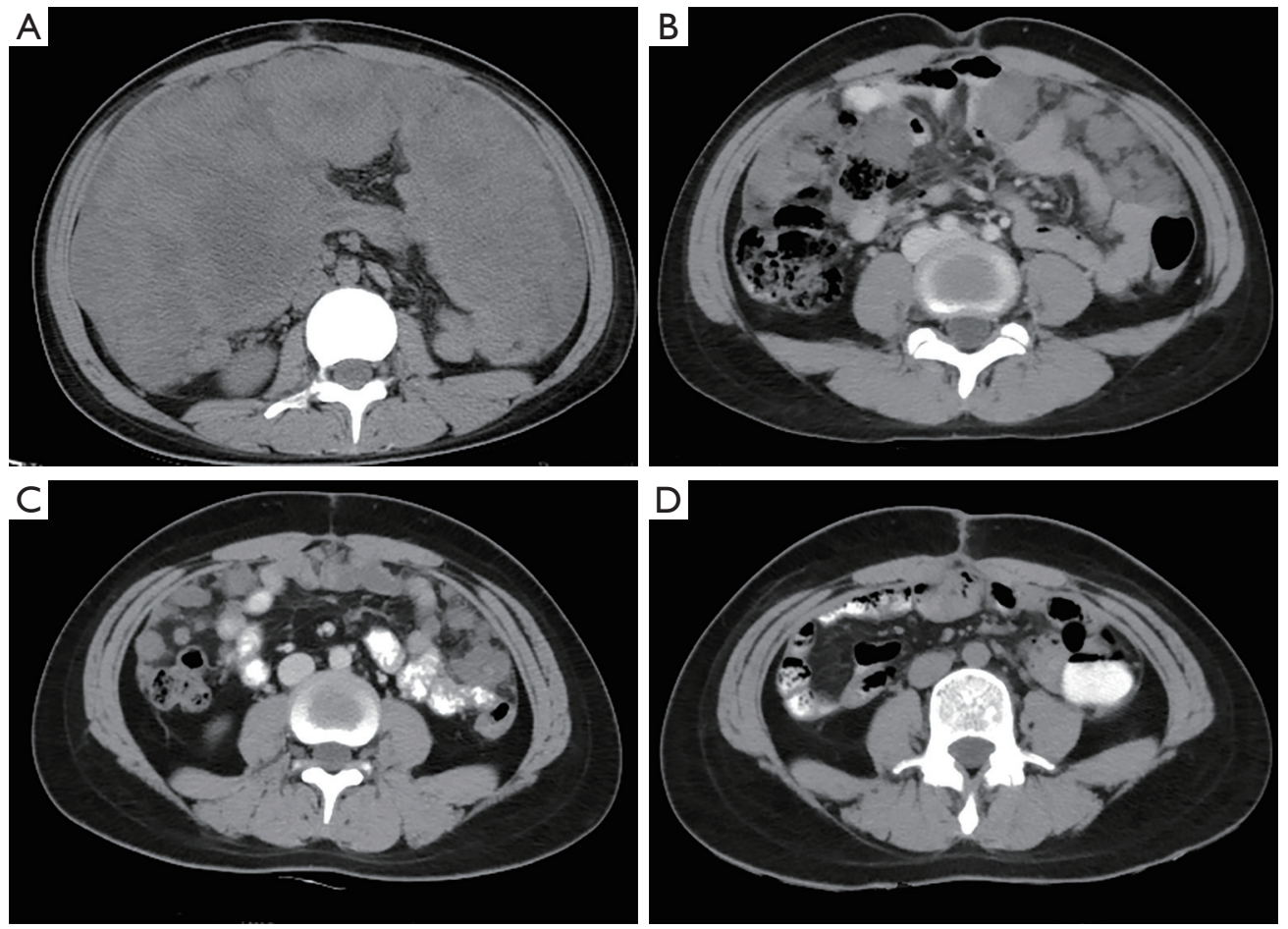

Figure 3 At first follow-up, there were extensively thickened masses and nodules of the peritoneum, omentum, and mesentery on abdominal computed tomography (CT) images (A). They gradually shrunk and disappeared at the second (B), third (C), and fourth follow-ups (D).

abdominal distension. Abdominal computed tomography (CT) examinations showed extensively thickened masses and nodules of the peritoneum, omentum, and mesentery (Figure $3 A$ ). The AFP level was up to $24,440 \mathrm{ng} / \mathrm{mL}$. It was confirmed as recurrence. Then, the patient was given 5 cycles of BEP (bleomycin, etoposide, and cisplatin). Serum AFP decreased to a normal level $(4.13 \mathrm{ng} / \mathrm{mL})$ after the $4^{\text {th }}$ cycle. Simultaneously, the thickened peritoneal, omental, and mesenteric masses on CT gradually shrunk and disappeared at the $4^{\text {th }}$ cycle (Figure 3B,C,D). Regular followups were performed monthly, and there was no evidence of recurrence for 4 months after the chemotherapy.

\section{Discussion}

YST is a teratoid neoplasm that can selectively differentiate towards vitelline (yolk sac) structures and undifferentiated embryonal cells in the tumor. It can present in the ovaries, testes, or extragonadal organs such as the mediastinum and the sacrococcygeal region $(6,7)$. As the second most common MOGCT, YST accounts for approximately $1 \%$ of all ovarian malignancies. It often occurs in young women or girls between the ages of 18 and 30 years old, having a high potential for malignancy, recurrence, and a poor prognosis $(6,8-10)$. DSD is a rare cause of intersexuality disorders, and is characterized by inconsistencies in the gonadal phenotype, gonadal anatomy, and chromosomal karyotype. It usually presents with ambiguous genitalia, such as a microphallus, urogenital sinus, hypospadias, and cryptorchidism. Its incidence is approximately $1 / 5,000$ to $1 / 4,500(3,4,11,12)$. The risk of malignancy in DSD is rare, though the most common neoplasm is germ cell tumor (GCT), and the most common histological type is dysgerminoma $(4,5)$. The occurrence of neoplasia is estimated to be $2.6 \%$ in patients with ovotesticular DSD, which was previously referred to as true hermaphroditism, and has a lower risk for the development of GCT in patients with the XX karyotype (11). Although YST and DSD are rare, here we present a case of a 23-year-old DSD patient with an ovarian YST. In this case, the patient underwent surgery but had a recurrence 2 months later, having now achieved clinical remission after 5 cycles of effective chemotherapy.

Elevated AFP is found in almost all patients with YST at diagnosis and is useful for the management and monitoring 
of YST remission or recurrence $(1,2)$. In this case, the preoperative APF level $(45,470 \mathrm{ng} / \mathrm{mL})$ was highly elevated. The patient had recurrence 2 months after surgery, and their AFP level was up to $24,440 \mathrm{ng} / \mathrm{mL}$. The abdominal CT images showed extensively thickened nodules and masses of the peritoneum, omentum, and mesentery. The patient had both high levels of AFP before surgery and recurrence, and these results are consistent with the literature $(1,2)$. Although YST is highly malignant with a high potential of recurrence, it is sensitive to chemotherapy (13). When this patient began chemotherapy, their AFP levels gradually decreased to normal, and the thickened nodules and masses of the peritoneum, omentum, and mesentery on CT scans also gradually shrunk and disappeared. Eventually, the patient achieved clinical remission after 5 cycles of chemotherapy treatment. This is consistent with previous literature demonstrating that YST has the characteristics of elevated AFP, recurrence, and sensitivity to chemotherapy treatment $(2,11)$.

Ovarian YST is usually large on presentation, and the maximum diameter can be up to $50 \mathrm{~cm}$. The tumor can be accompanied hemorrhage and necrosis in the solid tissue on the pathological cut section but is rarely unicystic or uniformly multicystic (2). It is not easy to diagnose clinically or to use radiology pre-operation. Although it is difficult to distinguish YST from other ovarian masses preoperatively by radiology, imaging tools can demonstrate a relationship between the tumor and its adjacent tissues and distant metastasis. In particular, MRI can reveal the hemorrhagic and hyper-vascularized features of the tumor $(2,9)$. In this case, the tumor presented with a hemorrhage and necrosis on the pathological cut section, and its MRI imaging also showed the hemorrhagic signal of hyperintensity on T1WI and hypervascular features on the enhanced phases. This suggests that MRI is a useful tool to demonstrate the tumor features and that AFP level, together with the hemorrhagic and hypervascularized features of the tumor on imaging, might help diagnose YST.

Abnormal gonadal development increases the risk of neoplasms, while DSD has been known as one of the main risk factors for developing type II GCTs, namely, dysgerminomas/seminomas and non-seminomas (e.g., YST, embryonal carcinoma, choriocarcinoma, and teratoma) $(11,14,15)$. In 1994, Nam et al. (14) reported a case of endodermal sinus tumor arising from a dysgenetic gonad in a 46, XX female patient. The patient presented with a huge mass approximately $17 * 15 \mathrm{~cm}$ from the right ovary that filled the entire abdominal and pelvic cavity and was accompanied by numerous metastatic nodules on the peritoneum and omentum. She also had marked elevation of AFP before surgery and a rising AFP level during disease progression. Ono et al. (16) reported another case of ovarian endodermal sinus tumor in a 15 -year-old girl with 45 , $\mathrm{XO} / 46, \mathrm{X}, \operatorname{dic}(\mathrm{Y})$ mosaicism. The patient's external genitalia was female; her left gonad consisted of a solid/cystic tumor (14 $\mathrm{cm}$ in diameter). Simultaneously, the right ovary was replaced by a streak that did not have any suggestion of testicular or ovarian tissue on microscopic examination, and her uterus was immature. The patient also had a high preoperative level of AFP, and her AFP level went back to normal after a total of 6 courses of chemotherapy for 1 year after surgery, having no signs of recurrence or metastasis in the follow-up time. To the best of our knowledge, there are no previous reports relating to DSD complicated by ovarian YST, especially in the 46, XX, male patient. Although the literature is limited, the patient, in this case, also had the same characteristics of elevated AFP, recurrence, and sensitivity to chemotherapy.

The risk of developing type II GCTs increases in DSD patients $(14,15)$. The importance of removing gonads in patients with gonadal dysgenesis, especially in patients with the $\mathrm{Y}$ chromosome, has been highlighted in previous reports (16). However, the risk of malignancy is low, and prophylactic removal of the gonad is not indicated in DSD $(4,5)$. Therefore, some controversy exists in regards to treatment. Furthermore, due to the scarcity of ovarian YSTs in DSD patients, there is no established standard treatment up to now, and management is challenging. A patient's special physiological structures, sex assignment, preservation of reproductive function, and achievement of long-term survival should all be considered. Given this complexity, its management is complicated and requires multidisciplinary team cooperation, including clinical geneticists, histopathologists, gynecologists, pediatric urologists, pediatric endocrinologists, pediatricians, psychiatrists or psychologists, and neonatologists $(3,11)$. Multi-center studies and international cooperation are also necessary (11). Although reports of DSD patients complicated by ovarian YSTs are limited, this case suggests that YST in DSD patients is also accompanied by elevated AFP, hemorrhage and hypervascularization features, recurrence, and sensitivity to chemotherapy. Chemotherapy combined with surgery may be a possible treatment for ovarian YST in patients with DSD and recurrence. Furthermore, to focus 
on the early detection of tumor recurrence and timely treatment, patients should have regular follow-ups and close monitoring of tumor signs after treatment.

As a case report, our findings provide a limited contribution regarding the imaging features, therapy, and prognosis of ovarian YST in DSD. However, this case suggested that ovarian YST in DSD also had the basic features of ovarian YSTs in females. Furthermore, it also proposed many important management issues, such as gender, fertility, and mental health, which should be thoroughly discussed and considered. Because of its rarity, no standardized treatment has been established for ovarian YST in DSD. Further studies are required to enhance understanding of this disease and its management.

\section{Acknowledgments}

The authors thank the numerous individuals who participated in this study, the editors, and the anonymous reviewers for their insightful suggestions on this work.

Funding: None.

\section{Footnote}

Conflicts of Interest: All authors have completed the ICMJE uniform disclosure form (available at http://dx.doi. org/10.21037/qims-20-890). The authors have no conflicts of interest to declare.

Ethical Statement: The ethics committee approved the study of Sichuan Cancer Hospital and Institute, Sichuan Cancer Center, School of Medicine, University of Electronic Science and Technology of China. All procedures performed in studies involving human participants were following the ethical standards of the institutional and/or national research committee(s). Written informed consent was obtained from the patient.

Open Access Statement: This is an Open Access article distributed in accordance with the Creative Commons Attribution-NonCommercial-NoDerivs 4.0 International License (CC BY-NC-ND 4.0), which permits the noncommercial replication and distribution of the article with the strict proviso that no changes or edits are made and the original work is properly cited (including links to both the formal publication through the relevant DOI and the license). See: https://creativecommons.org/licenses/by-nc-nd/4.0/.

\section{References}

1. Nasioudis D, Chapman-Davis E, Frey MK, Caputo TA, Holcomb K. Management and prognosis of ovarian yolk sac tumors; an analysis of the National Cancer Data Base. Gynecol Oncol 2017;147:296-301.

2. Dällenbach P, Bonnefoi H, Pelte MF, Vlastos G. Yolk sac tumours of the ovary: an update. Eur J Surg Oncol 2006;32:1063-75.

3. Tranoulis A, Michala L. Hermaphroditism: an obsolete diagnosis. Hormones (Athens) 2017;16:104-6.

4. Montero M, Méndez R, Valverde D, Fernández JL, Gómez M, Ruíz C. True hermaphroditism and normal male external genitalia: a rare presentation. Acta Paediatr 1999;88:909-11.

5. Ceci M, Calleja E, Said E, Gatt N. A case of true hermaphroditism presenting as a testicular tumour. Case Rep Urol 2015;2015:598138.

6. Kao CS, Idrees MT, Young RH, Ulbright TM. Solid pattern yolk sac tumor: a morphologic and immunohistochemical study of 52 cases. Am J Surg Pathol 2012;36:360-7.

7. Shah JP, Kumar S, Bryant CS, Ali-Fehmi R, Malone JM Jr, Deppe G, Morris RT. A population-based analysis of 788 cases of yolk sac tumors: A comparison of males and females. Int J Cancer 2008;123:2671-5.

8. Kojimahara T, Nakahara K, Takano T, Yaegashi N, Nishiyama H, Fujimori K, Sato N, Terada Y, Tase T, Yokoyama Y, Mizunuma H, Shoji T, Sugiyama T, Kurachi H.. Yolk sac tumor of the ovary: a retrospective multicenter study of 33 Japanese women by Tohoku Gynecologic Cancer Unit (TGCU). Tohoku J Exp Med 2013;230:211-7.

9. Chen LH, Yip KC, Wu HJ, Yong SB. Yolk Sac Tumor in an Eight-Year-Old Girl: A Case Report and Literature Review. Front Pediatr 2019;7:169.

10. Tao T, Yang JX, Cao DY, Liu JT, Shen K. Management of recurrent endodermal sinus tumor of the ovary associated with pregnancy. Gynecol Oncol 2011;122:455-6.

11. Pleskacova J, Hersmus R, Oosterhuis JW, Setyawati BA, Faradz SM, Cools M, Wolffenbuttel KP, Lebl J, Drop SL, Looijenga LH. Tumor risk in disorders of sex development. Sex Dev 2010;4:259-69.

12. Lee PA, Nordenström A, Houk CP, Ahmed SF, Auchus R, Baratz A, Baratz Dalke K, Liao LM, Lin-Su K, Looijenga LH 3rd, Mazur T, Meyer-Bahlburg HF, Mouriquand P, Quigley CA, Sandberg DE, Vilain E, Witchel S; Global 
DSD Update Consortium. Global Disorders of Sex

Development Update since 2006: Perceptions, Approach and Care. Horm Res Paediatr 2016;85:158-80.

13. Chang YW, Chao KC, Sung PL, Li WH, Wang PH.

Treatment of ovarian endodermal sinus tumor to preserve fertility. J Chin Med Assoc 2013;76:112-4.

14. Nam JH, Chung DG, Kim YM, Kim YT, Mok JE.

Endodermal sinus tumor arising from a dysgenetic gonad in a 46, XX female combined with müllerian agenesis.

Cite this article as: Xie CL, Peng CR, Yan JX, Wang LX, Zhou P. Ovarian yolk sac tumor in a patient with sexual differentiation disorder: a case description. Quant Imaging Med Surg 2021;11(7):3360-3366. doi: 10.21037/qims-20-890
Gynecol Oncol 1994;55:465-8.

15. Looijenga LH, Hersmus R, de Leeuw BH, Stoop H, Cools M, Oosterhuis JW, Drop SL, Wolffenbuttel KP. Gonadal tumours and DSD. Best Pract Res Clin Endocrinol Metab 2010;24:291-310.

16. Ono T, Sakai N, Hayashi Y, Saito M, Kawagoe S, Hiroi M. 45, XO/46, X, dic (Yq) mosaicism in Turner's phenotype with endodermal sinus tumor of the ovary. Gynecol Obstet Invest 1989;27:45-7. 